\title{
Single-Entity Heparan Sulfate Glycomimetic Clusters for Therapeutic Applications**
}

\author{
Peter C. Tyler, Scott E. Guimond, Jeremy E. Turnbull, and Olga V. Zubkova*
}

Dedicated to Professor Richard H. Furneaux on the occasion of his 65th birthday

\begin{abstract}
Heparan sulfate (HS) is a highly sulfated glycosaminoglycan with a variety of critical functions in cell signaling and regulation. HS oligosaccharides can mimic or interfere with HS functions in biological systems; however, their exploitation has been hindered by the complexity of their synthesis. Polyvalent displays of small specific HS structures on dendritic cores offer more accessible constructs with potential advantages as therapeutics, but the synthesis of single-entity $H S$ polyvalent compounds has not previously been described. Herein we report the synthesis of a novel targeted library of single-entity glycomimetic clusters capped with varied $H S$ saccharides. They have the ability to mimic longer natural HS saccharides in their inhibition of the Alzheimer's disease (AD) protease BACE-1. We have identified several single-entity $H S$ clusters with $I C_{50}$ values in the low-nanomolar range. These HS clusters are drug leads for $A D$ and offer a novel framework for the manipulation of heparan sulfate-protein interactions in general.
\end{abstract}

$\boldsymbol{H}_{\mathrm{e}}$ eparan sulfate (HS) and heparin belong to the glycosaminoglycan family. These linear polysaccharides are made of repeating disaccharide units, which can be differentially sulfated at the $2-\mathrm{O}$ position of the D-glucuronic acid or Liduronic acid and/or the 6-O and, rarely, 3-O positions of the glucosamine. The glucosamine amino group can be $\mathrm{N}$-sulfated and $\mathrm{N}$-acetylated. The multiple biological functions of $\mathrm{HS}$ are due to ionic interactions between negatively charged sulfates and carboxylate groups with a variety of proteins, such as growth factors, ${ }^{[1]}$ proteases, ${ }^{[2]}$ and cytokines. ${ }^{[3]}$ HS plays crucial roles in cancer, ${ }^{[4]}$ inflammation, ${ }^{[5]}$ bone repair, ${ }^{[6]}$ angiogenesis, ${ }^{[7]}$ anticoagulation, ${ }^{[8]}$ viral invasion, ${ }^{[9]}$ and neurodegenera-

[*] Dr. P. C. Tyler, Dr. O. V. Zubkova

The Ferrier Research Institute, Victoria University of Wellington

Gracefield Research Centre

Lower Hutt (New Zealand)

E-mail: Olga.Zubkova@vuw.ac.nz

Dr. S. E. Guimond, Prof. J. E. Turnbull

Institute of Integrative Biology, University of Liverpool

Liverpool L69 3BX (UK)

[**] We acknowledge Dr. Herbert Wong and Dr. Yinrong Lu for an excellent NMR and mass spectrometry service. We thank Dr. Ralf Schwörer for the synthesis of a monosaccharide intermediate, and Eizabeth Edwards for technical assistance with the bioassays. J.E.T. gratefully acknowledges the financial support of the Biotechnology and Biological Sciences Research Council UK and the Medical Research Council UK.

D. Supporting information for this article is available on the WWW under http://dx.doi.org/10.1002/anie.201410251. tive disorders. ${ }^{[10]}$ However, the potentially numerous therapeutic applications of HS oligosaccharides have not been realized, largely because of the difficulty and complexity of the synthesis of these compounds. Fondaparinux (Arixtra), a pharmaceutical anticoagulant, is an example of a synthetic sulfated oligosaccharide. ${ }^{[11]}$ However, the synthetic route to this compound involves more than 40 steps.

A decade ago, HS was identified as a natural regulator of the protease $\beta$-secretase (BACE- 1 ), which generates toxic $\beta$ amyloid $(A \beta)$ peptides as products; these peptides aggregate into plaques in the brains of patients with Alzheimer's disease (AD). ${ }^{[12]}$ Inhibitors of BACE-1 are expected to block this process, thus providing a disease-modifying treatment by preventing the formation of plaques and the degeneration of neurons in the disease process.

Recently, we developed a novel synthetic approach to HS oligosaccharides and prepared a targeted library of HS fragments (ranging in size from 6- to 12 -mers). ${ }^{[13]}$ We have shown these compounds to be promising BACE-1 inhibitors with low-nanomolar $\mathrm{IC}_{50}$ values that could be used to prevent the accumulation of $\mathrm{A} \beta$ peptides. The complex synthesis of HS oligosaccharides has been investigated by a number of research groups ${ }^{[14]}$ but despite many useful modifications and improved glycosylation protocols, the syntheses of HS targets still require multiple steps ( $>50$ steps $)^{[15]}$ and remain cumbersome and costly. In this study, with the aim of greatly simplifying the synthetic process while retaining the desired bioactivity of target HS structures, we decorated tetravalent dendritic cores with multiple short, readily synthesized HS sequences (Scheme 1).

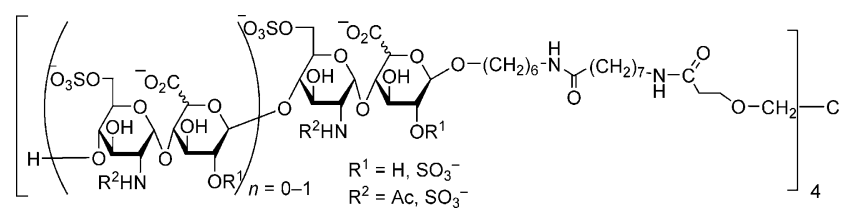

Scheme 1. Synthetic targets.

Dendritic cores ${ }^{[16]}$ and dendrimers ${ }^{[17]}$ provide a framework to exploit polyvalency, a key principle in nature for the establishment of strong yet reversible "Velcro"-type interactions for biomolecular recognition. ${ }^{[18]}$ Traditional drug discovery has focused on small-molecule drugs that attach to a single binding site $;^{[19]}$ however, biological targets are large molecules which often rely on polyvalent interactions at multiple sites in their binding cascades. ${ }^{[20]}$ The weak inter- 
actions of individual subunit structures are often enhanced by their specific repetition, known as a synergistic clustering effect. ${ }^{[21]}$ However, many polyvalent compounds and polymers synthesized for potential therapeutic applications are not single chemical entities and it is difficult to manufacture such mixtures for FDA approval. Several studies have been directed towards the synthesis of HS glycomimetics. The multivalent presentation of heparin hexasaccharides on PAMAM (polyamidoamine) dendrimers furnished potent fibroblast growth factor (FGF) regulators and demonstrated the ability of hyperbranched macromolecules to amplify the binding affinity of heparin fragments. However, the inability to fully cap the dendrimers led to mixtures of glycoconjugates. $^{[22]}$ A synthesis of selectively sulfated HS glycopolymers of controllable length was recently reported for the targeting of chemokines and their receptor interactions, but the products were mixtures of polymers decorated with heparin fragments. ${ }^{[23]}$ Herein we report the synthesis of single-entity tetravalent glycomimetics end-capped with short specific HS sequences and their ability to mimic much longer polyand oligosaccharide chains of natural HS in their inhibition of BACE-1.

A Michael reaction of pentaerythritol (1) with acrylonitrile, followed by treatment with concentrated $\mathrm{HCl}$, gave the known tetraacid $\mathbf{2}^{[24]}$ the treatment of which with $\mathrm{N}$ hydroxysuccinimide (NHS) and 1-ethyl-3-(3-dimethylaminopropyl)carbodiimide (EDC) afforded the "short-armed" tetra- $N$-hydroxysuccinimide-activated ester 3 as a crystalline material. 8-Aminooctanoic acid (4) was converted into the benzyl ester $5^{[25]}$ and coupled with 3 to give the tetravalent dendritic core $\mathbf{6}$. Hydrogenolysis of 6 gave the tetraacid 7, the treatment of which with NHS/EDC furnished the "long-armed" $N$-hydroxysuccinimide ester 8 (Scheme 2a).

To evaluate the coupling methodology, we prepared two model glycomimetic clusters, 9 and $\mathbf{1 0}$ (Scheme 2b). The sixcarbon-atom linker $\mathbf{1 1}$ was attached to the glucosamine building block $\mathbf{1 2}^{[13]}$ to give $\mathbf{1 3}$, which was converted into the $\mathrm{N}$-acetate 14. Zemplen deacetylation followed by sulfation 
and unmasking of the amino functionality by hydrogenolysis afforded the sulfated monomer 15. Coupling of $\mathbf{1 5}$ to the short-armed active ester core $\mathbf{3}$ led to complex mixtures of starting material, some di- and trisubstituted clusters, and the tetrasubstituted cluster $\mathbf{9}$, all of which had similar $R_{\mathrm{f}}$ values on TLC plates. Repeated chromatography on silica gel produced 9 in $40 \%$ yield. We reasoned that electrostatic repulsion of anionic sulfo groups led to only partial substitution and formation of the product 9 in moderate yield. In an attempt to improve the coupling yield, we attached the fully protected monosaccharide $\mathbf{1 6}$ to the dendritic core $\mathbf{3}$. This reaction successfully afforded cluster $\mathbf{1 7}$ in almost quantitative yield. Deacetylation followed by O-sulfation furnished a "shortarmed" cluster 19. Hydrogenolysis then gave a pure cluster 9 in excellent yield (Scheme 2 b). Encouraged by these results, we prepared a "long-armed" tetramer cluster $\mathbf{1 0}$ in the same manner. Again, the coupling yield, in this case for the formation of the tetramer $\mathbf{2 0}$, was almost quantitative. Derivatization steps gave the sulfated cluster $\mathbf{1 0}$ as a pure single product.

When assessed as inhibitors of BACE-1, the potency of these compounds clearly increased from the monomer $\mathbf{1 5}$ to the "short-armed" cluster $\mathbf{9}$ to the "long-armed" cluster $\mathbf{1 0}$ (Table 1). For this reason, the long-armed dendritic core was used to prepare a targeted library of HS glycomimetics.

afforded only the fully protected tetrasaccharides $\mathbf{3 5}$ and $\mathbf{3 6}$, respectively, in 85 and $87 \%$ yield (Scheme 3). These di- and tetrasaccharides were then partially processed to a stage suitable for coupling to the cores. Selective removal of the chloroacetate groups and oxidation of the resulting primary hydroxy groups in 37-40, followed by ester formation, provided the methyl esters $\mathbf{4 1 - 4 4}$ in excellent yield. Treatment with thioacetic acid in pyridine then produced the $\mathrm{N}$-acetates 45-47, and the linker amino groups were unmasked by treatment with $\mathrm{Zn}$ in $\mathrm{AcOH}$ ( $N$-Troc) or by hydrogenolysis $(\mathrm{N}-\mathrm{Cbz})$ to afford the fully protected $\mathrm{N}$-acetylated di- and tetrasaccharide HS fragments 48-50. The $N$-Fmoc group in $\mathbf{4 4}$ was removed selectively with piperidine to furnish the tetrasaccharide 51. The sulfated disaccharide monomer 54 was prepared from $\mathbf{4 6}$ in three steps: Zemplen deacetylation, selective O-sulfation, and brief hydrogenolysis. Compound $\mathbf{5 4}$ was compared to the tetravalent compounds as BACE1 inhibitors to assess the clustering effect in these glycomimetics.

Coupling of four equivalents of the $N$-acetylated HS fragments $\mathbf{4 8}-\mathbf{5 1}$ with the tetra- $N$-hydroxysuccinimide active ester $\mathbf{8}$ afforded tetramer clusters 55-58 in $93-95 \%$ yield after chromatography (Scheme 4). Acidic hydrolysis selectively removed the $O$-acetate groups to give 59-62 in high yield, and sulfation of the primary hydroxy groups furnished 63-66, which were purified by chromatography. Simultaneous saponification of the benzoate and methyl esters then generated tetramers 67-70. Hydrogenolysis of 67-69 in aqueous THF basified with aqueous ammonia gave the targeted 6-O-sulfated $\mathrm{N}$-acetylated tetramers $\mathbf{7 1 - 7 3}$, which were isolated as their sodium salts. Sulfation of the tetramer clusters 67-70 afforded 74-77. Hydrogenolysis then furnished the targeted $N$-acetylated tetramers $\mathbf{7 8}$ 80 with two sulfate groups per disaccharide unit as well as the amino tetramer 81. N-Sulfation of 81 under aqueous conditions gave the desired octa-N-sulfated hexadeca-O-sulfated HS tetramer $\mathbf{8 2}$.

The ability of the target compounds to inhibit the BACE-1 pro-

We previously prepared a library of hexa- to dodecasaccharide fragments of $\mathrm{HS}$ and showed that 6-O-sulfation of the GlcNAc residues was essential for BACE-1 inhibition, whereas 2-O-sulfation of the uronic acids (UAs) increased activity (especially in shorter saccharides, such as octasaccharides) and $\mathrm{N}$-acetylation was well-tolerated. ${ }^{[13]}$ These results provided a template for the HS structures to cap the tetravalent cores.

The glycosylation of donors $\mathbf{2 3}-\mathbf{2 6}{ }^{[13]}$ variously with the linkers 11, 27, and $\mathbf{2 8}$ afforded only the $\beta$-disaccharides $\mathbf{2 9}, \mathbf{3 0}$, 33, and $\mathbf{3 4}$ in $80-90 \%$ yield (Scheme 3 ). The $O$-Fmocprotected derivatives $\mathbf{2 9}$ and $\mathbf{3 0}$ were converted into acceptors $\mathbf{3 1}$ and 32, and then coupling of 25 with $\mathbf{3 1}$ and $\mathbf{2 6}$ with 32 tease was next investigated by the use of fluorescence resonance energy transfer (FRET) peptide-cleavage assays (see the Supporting Information). All glycomimetic clusters inhibited BACE-1 with $\mathrm{IC}_{50}$ values in the micromolar to lownanomolar range (Table 1). Tetramers 9 and $\mathbf{1 0}$ with $\mathrm{O}$ sulfated GlcNAc (GlcNAc6S) attached showed low potency. We found that the potency increased from an $\mathrm{IC}_{50}$ value of $25 \mu \mathrm{m}$ for the sulfated "short-armed" small cluster 9 to a value of $0.76 \mu \mathrm{m}$ for the larger "long-armed" cluster $\mathbf{1 0}$. The clusters with 6-O-sulfated GlcNAc in disaccharide fragments (GlcNAc6S-UA) $\mathbf{7 1}$ and $\mathbf{7 2}$ were only micromolar inhibitors, and the presence of D-glucuronic acid or L-iduronic acid as the end-capping saccharide led to no difference in potency. 


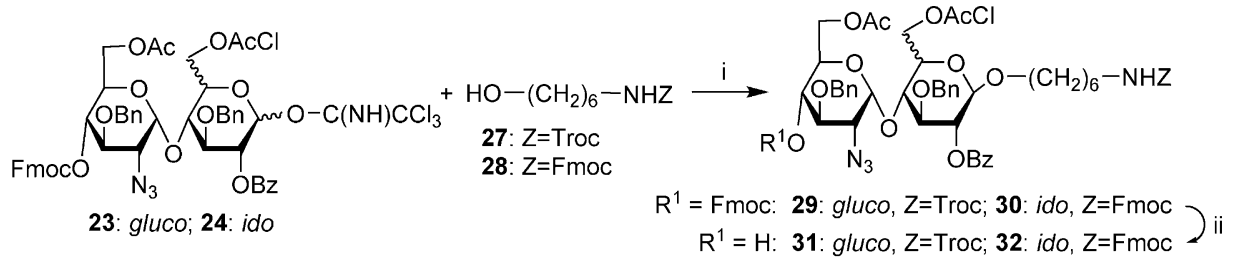

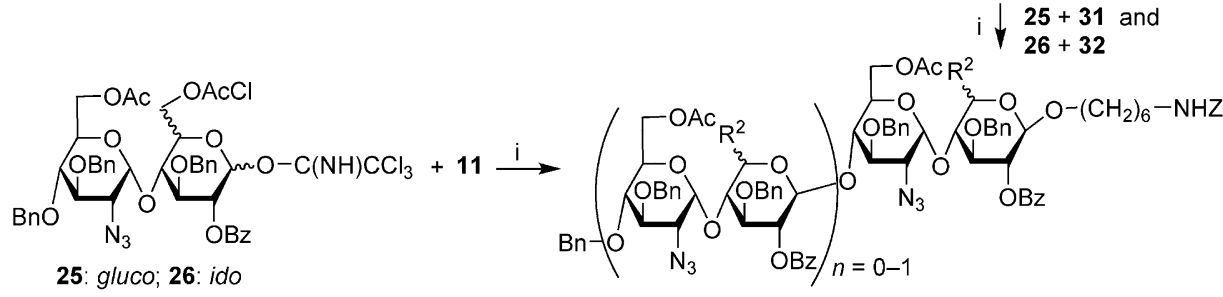

iii $\mathrm{R}^{2}=\mathrm{CH}_{2} \mathrm{OCOCH}_{2} \mathrm{Cl}: 33: n=0$, gluco, $\mathrm{Z}=\mathrm{Cbz} ; 34: n=0, i d o, \mathrm{Z}=\mathrm{Cbz}$ (ii $\mathrm{R}^{2}=\mathrm{CH}_{2} \mathrm{OH}: 37$ : $n=1$, gluco, $\mathrm{Z}=$ Troc; 36: $n=1$, ido, $\mathrm{Z}=\mathrm{Fmoc}$ , gluco, $Z=\mathrm{Cbz} ; 38: n=0$, ido, $Z=\mathrm{Cbz}$ 39: $n=1$, gluco, Z=Troc; $\mathbf{4 0}: n=1, i d o, Z=F m o c$

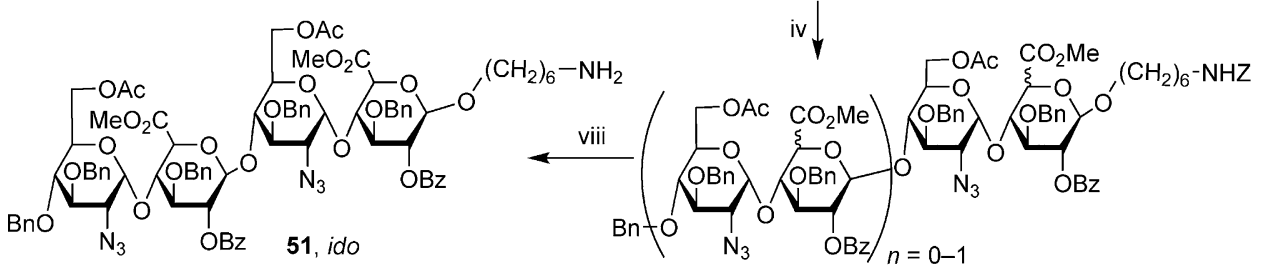

41: $n=0$, gluco, $Z=\mathrm{Cbz} ; 42: n=0$, ido, $Z=\mathrm{Cbz}$ 43: $n=1$, gluco, $Z=$ Troc; $44: n=1$, ido, $Z=F m o c$
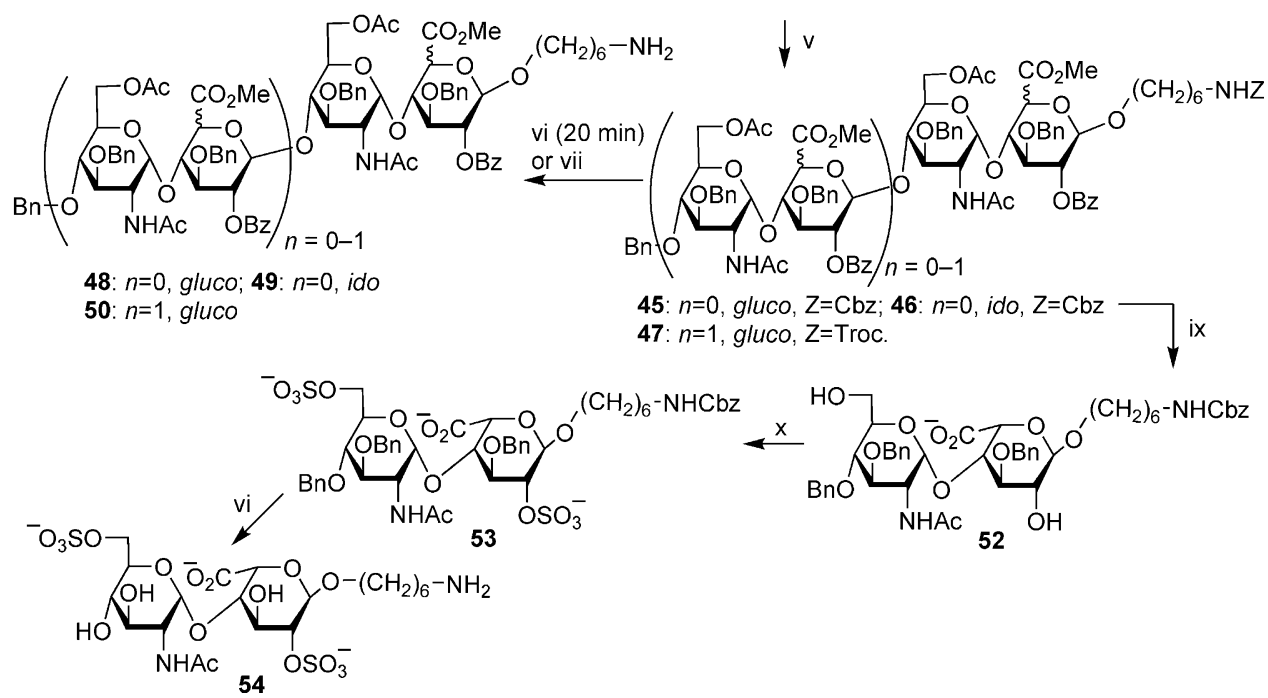

Scheme 3. Synthesis of di- and tetrasaccharide building blocks with linkers. i) TMSOTf, $\mathrm{CH}_{2} \mathrm{Cl}_{2},-30^{\circ} \mathrm{C} \rightarrow$ $\mathrm{RT}$; ii) $\mathrm{Et}_{3} \mathrm{~N}, \mathrm{CH}_{2} \mathrm{Cl}_{2}$, room temperature; iii) $\mathrm{DABCO}, \mathrm{MeCN} / \mathrm{EtOH}, 70^{\circ} \mathrm{C}$; iv) TEMPO, $\mathrm{BAIB}$, aqueous $\mathrm{MeCN}$, room temperature, then $\mathrm{CH}_{2} \mathrm{~N}_{2}, 0^{\circ} \mathrm{C} \rightarrow \mathrm{RT}$; v) $\mathrm{HSAc}$, pyridine, room temperature; vi) $\mathrm{H}_{2}, \mathrm{Pd}(\mathrm{OH})_{2} / \mathrm{C}$, aqueous $\mathrm{MeOH}$, room temperature; vii) $\mathrm{Zn}, \mathrm{AcOH}$, THF, room temperature; viii) piperidine, DMF, room temperature; ix) $\mathrm{NaOH}$, aqueous $\mathrm{MeOH}, 0^{\circ} \mathrm{C} \rightarrow \mathrm{RT}$; x) $\mathrm{SO}_{3} \cdot \mathrm{NMe}_{3}, \mathrm{DMF}, 60^{\circ} \mathrm{C}$. The descriptors ido/gluco are used to describe the configuration of the carbohydrate residues representing uronic acids in the target molecule (ido-for a (GlcNAc-IdoA) $)_{n=0-1}$ and gluco- for a (GlcNAc-GlcA $)_{n=0-1}$ saccharide). BAIB = bis(acetoxy) iodobenzene, $\mathrm{Bz}=$ benzoyl, $\mathrm{DABCO}=1,4$-diazabicyclo[2.2.2] octane, $\mathrm{Fmoc}=$ fluorenyl-9-methoxycarbonyl, TEMPO $=(2,2,6,6$-tetramethylpiperidin-1-yl) oxy, TMS $=$ trimethylsilyl, Troc $=2,2,2$-trichloroethoxycarbonyl.

The potency increased when the 2-O position of the UA was sulfated (GlcNAc6S-UA2S); thus, tetramers $\mathbf{7 8}$ and $\mathbf{7 9}$ had $\mathrm{IC}_{50}$ values of 13 and $52 \mathrm{~nm}$. Tetramer 73 with larger HS saccharides attached, tetrasaccharides GlcNAc6S-GlcA-
GlcNAc6S-GlcA, displayed an $\mathrm{IC}_{50}$ value of $37 \mathrm{~nm}$. This bioactivity was increased with sulfation of the 2-O position of the UA: Inhibitor 80 capped with GlcNAc6S-GlcA2SGlcNAc6S-GlcA2S tetrasaccharides showed an $\mathrm{IC}_{50}$ value of $1.6 \mathrm{nM}$. It is notable that this potency is similar to that observed for the most potent 8-12-mer synthetic HS saccharides previously described as BACE-1 inhibitors. ${ }^{[13]}$ The Nsulfation of glucosamine units to produce GlcNS6S-IdoA2SGlcNS6S-IdoA2S capping saccharides in $\mathbf{8 2}$ led to only a slight increase in potency $\left(\mathrm{IC}_{50}=1.5 \mathrm{~nm}\right)$.

Importantly, none of the compounds displayed any significant anticoagulant activity towards factor $\mathrm{Xa}(<0.1 \%$ of the activity of unfractionated heparin). Furthermore, in regard to potential off-target effects, all compounds displayed very low abilities to inhibit the structurally related aspartyl protease cathepsin D; known inhibitors of this enzyme, such as pepstatin A, are potent $\left(\mathrm{IC}_{50} \approx 1 \mathrm{nM}\right)$, whereas the most inhibitory GlcNAc6S-containing cluster 80, the N-sulfated cluster $\mathbf{8 2}$, and heparin displayed $\mathrm{IC}_{50}$ values of $>1 \mu \mathrm{M}, 24 \mathrm{nM}$, and $3.3 \mathrm{~nm}$, respectively (see Table 2 in the Supporting Information). All compounds also proved incapable of activating fibroblast growth factor (FGF) signaling by either FGF-1 or FGF-2 through FGF receptor 1 at concentrations up to $10 \mu \mathrm{g} \mathrm{mL}^{-1}$, whereas heparin has $\mathrm{EC}_{50}$ values of 500 and $30 \mathrm{ng} \mathrm{mL}^{-1}$, respectively, in these assays (see Table 3 in the Supporting Information).

To summarize, we have for the first time prepared singleentity compounds presenting polyvalent displays of HS saccharides. We have shown that these clusters can mimic longer HS oligosaccharides in their ability to inhibit BACE-1, an Alzheimer's disease drug target, and lack off-target 


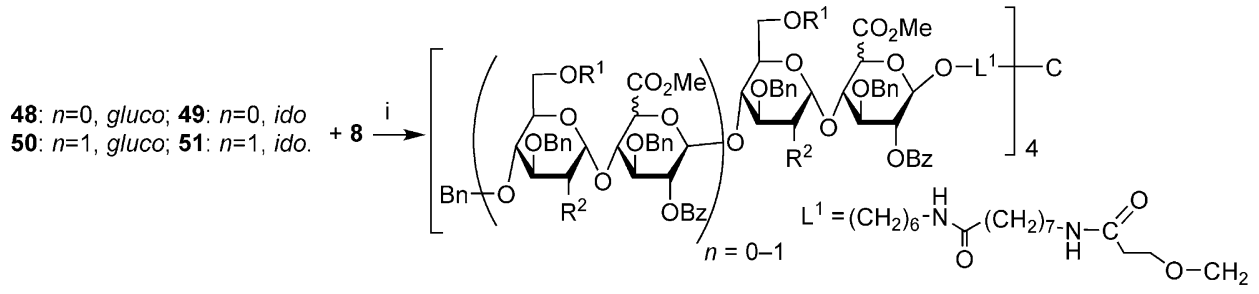

ii $\mathrm{R}^{1}=\mathrm{Ac}, \mathrm{R}^{2}=\mathrm{NHAc}: 55: n=0$, gluco; 56: $n=0$, ido; 57: $n=1$, gluco; $\mathrm{R}^{1}=\mathrm{Ac}, \mathrm{R}^{2}=\mathrm{N}_{3}: 58: n=1$, ido;

iii $\mathrm{R}^{1}=\mathrm{H}, \mathrm{R}^{2}=\mathrm{NHAc}: 59: n=0$, gluco; $60: n=0, i d o ; 61: n=1$, gluco; $\mathrm{R}^{1}=\mathrm{H}, \mathrm{R}^{2}=\mathrm{N}_{3}: \mathbf{6 2}: n=1$, ido;

iii $\mathrm{R}^{1}=\mathrm{SO}_{3}, \mathrm{R}^{2}=$ NHAc: 63: $n=0$, gluco; 64: $n=0$, ido; 65: $n=1$, gluco; $\mathrm{R}^{1}=\mathrm{SO}_{3}, \mathrm{R}^{2}=\mathrm{N}_{3}: 66: n=1$, ido

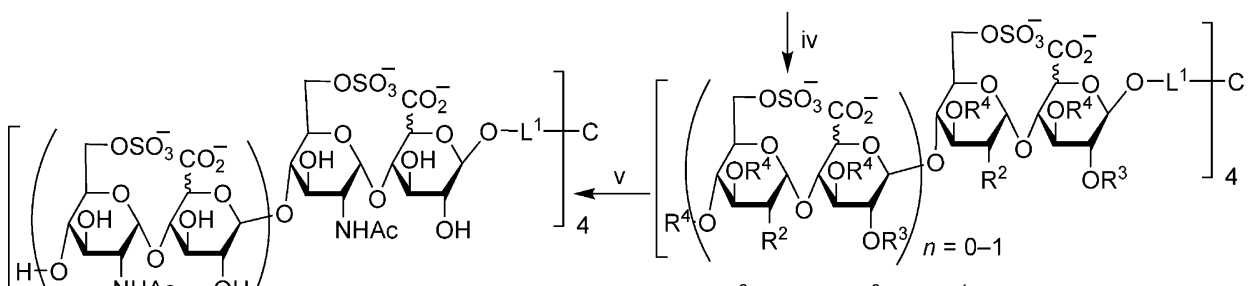

71: $n=0$, gluco; $72: n=0$, ido; $73: n=1$, gluco
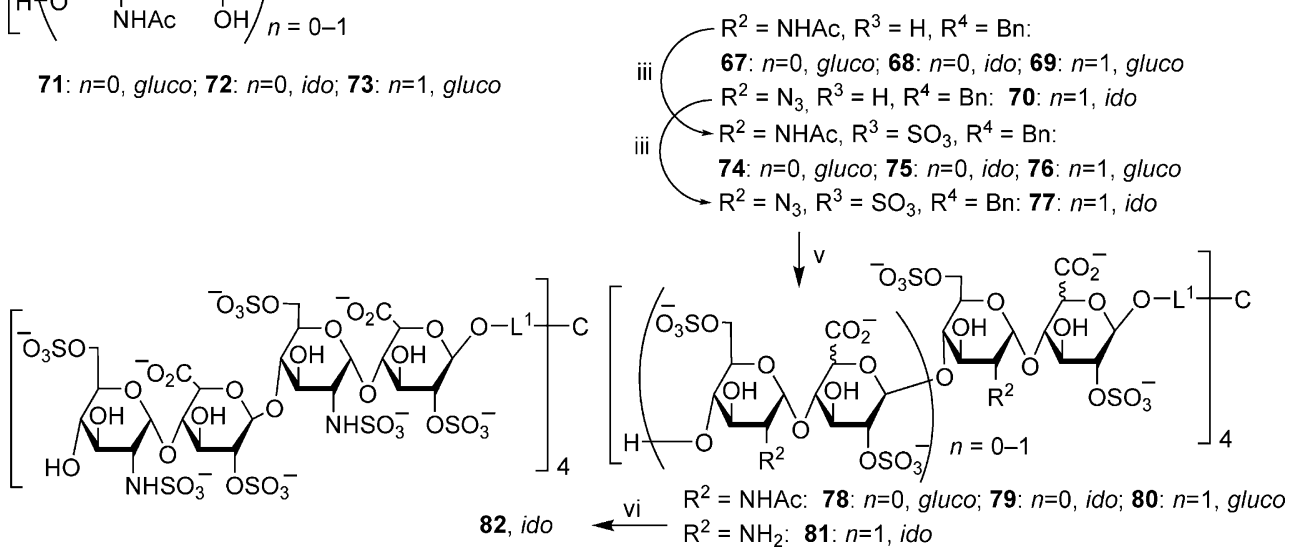

Scheme 4. Assembly of tetravalent $\mathrm{HS}$ clusters. i) $\mathrm{Et}_{3} \mathrm{~N}$, DMF, room temperature; ii) $\mathrm{HCl}, \mathrm{MeOH} / \mathrm{CH}_{2} \mathrm{Cl}_{2}$, $0^{\circ} \mathrm{C} \rightarrow \mathrm{RT}$; iii) $\mathrm{SO}_{3} \cdot \mathrm{NMe}_{3}, \mathrm{DMF}, 60^{\circ} \mathrm{C}$; iv) $\mathrm{NaOH}$, aqueous $\mathrm{MeOH}, 0^{\circ} \mathrm{C} \rightarrow \mathrm{RT}$; v) $\mathrm{H}_{2}, \mathrm{Pd}(\mathrm{OH})_{2} / \mathrm{C}$, aqueous THF, room temperature; vi) $\mathrm{SO}_{3} \cdot \mathrm{NMe}_{3}, \mathrm{Na}_{2} \mathrm{CO}_{3}, \mathrm{H}_{2} \mathrm{O}, 0^{\circ} \mathrm{C} \rightarrow \mathrm{RT}$.

anticoagulant activity. Most crucially, they are much more synthetically accessible than HS saccharides with equivalent BACE-1-inhibitory activity. The compounds reported herein are novel leads as drugs for AD. Dendritic cores and associated methodology provide a template for the polyvalent presentation of other HS saccharides optimized for other indications.

Received: October 19, 2014

Revised: December 1, 2014

Published online: January 13, 2015

Keywords: Alzheimer's disease - dendrimers - glycomimetics . heparan sulfate $\cdot$ oligosaccharides

[1] J. Turnbull, A. Powell, S. Guimond, Trends Cell Biol. 2001, 11, $75-82$.

[2] J. R. Bishop, M. Schuksz, J. D. Esko, Nature 2007, 446, $1030-$ 1037.

[3] H. Lortat-Jacob, Curr. Opin. Struct. Biol. 2009, 19, 543-548.

[4] N. Turner, R. Grose, Nat. Rev. Cancer 2010, 10, 116-129.

[5] C. R. Parish, Nat. Rev. Immunol. 2006, 6, 633-643.
[6] S. Murali, B. Rai, C. Dombrowski, J. L. J. Lee, Z.X. H. Lim, D. S. Bramono, L. Ling, T. Bell, S. Hinkley, S. S. Nathan, J. H. Hui, H. K. Wong, V. Nurcombe, S. M. Cool, Biomaterials 2013, 34, 5594-5605.

[7] M. M. Fuster, J. D. Esko, Nat. Rev. Cancer 2005, 5, $526-542$.

[8] R. Lever, C. P. Page, Nat. Rev. Drug Discovery 2002, $1,140-148$.

[9] M.-J. Oh, J. Akhtar, P. Desai, D. Shukla, Biochem. Biophys. Res. Commun. 2010, 391, 176-181.

[10] J. van Horssen, P. Wesseling, L. P. W. J. van den Heuvel, R. M. W. de Waal, M. M. Verbeek, Lancet Neurol. 2003, 2, 482-492.

[11] A. G. G. Turpie, Eur. Heart J. Suppl. 2008, 10, C1-C7.

[12] a) J. Hardy, D. J. Selkoe, Science 2002, 297, 353356; b) Z. Scholefield, E. A. Yates, G. Wayne, A. Amour, W. McDowell, J. E. Turnbull, J. Cell Biol. 2003, 163, 97-107.

[13] R. Schwörer, O. V. Zubkova, J. E. Turnbull, P. C. Tyler, Chem. Eur. J. 2013, 19, 6817-6823.

[14] a) H. A. Orgueira, A. Bartolozzi, P. Schell, R. E. J. N. Litjens, E. R. Palmacci, P. H. Seeberger, Chem. Eur. J. 2003, 9, 140-169; b) L.-D. Lu, C.-R. Shie, S. S. Kulkarni, G.-R. Pan, X.-A. Lu, S.-C. Hung, Org. Lett. 2006, 8, 5995-5998; c) K. A. Allen, R. L. Brown, G. Norris, P. C. Tyler, D. K. Watt, O. V. Zubkova, Carbohydr. Res. 2010, 345, $1831-1841$

[15] a) T. Polat, C.-H. Wong, J. Am. Chem. Soc. 2007, 129, 1279512800 ; b) K. Yoshida, B. Yang, W. Yang, Z. Zhang, J. Zhang, X. Huang, Angew. Chem. Int. Ed. 2014, 53, 9051-9058; Angew. Chem. 2014, 126, 9197-9204; c) Y. Chen, Y. Li, H. Yu, G. Sugiarto, V. Thon, J. Hwang, L. Ding, L. Hie, X. Chen, Angew. Chem. Int. Ed. 2013, 52, 11852-11856; Angew. Chem. 2013, 125, $12068-12072$.

[16] G. R. Newkome, A. Mishra, C. N. Moorefield, J. Org. Chem. 2002, 67, 3957-3960

[17] a) D. A. Tomalia, A. M. Naylor, W. A. Goddard, Angew. Chem. Int. Ed. Engl. 1990, 29, 138-175; Angew. Chem. 1990, 102, 119157; b) R. J. Amir, N. Pessah, M. Shamis, D. Shabat, Angew. Chem. Int. Ed. 2003, 42, 4494-4499; Angew. Chem. 2003, 115, $4632-4637$.

[18] C. Fasting, C. A. Schalley, M. Weber, O. Seitz, S. Hecht, B. Koksch, J. Dernedde, C. Graf, E.-W. Knapp, R. Haag, Angew. Chem. Int. Ed. 2012, 51, 10472-10498; Angew. Chem. 2012, 124, $10622-10650$.

[19] a) C. A. Lipinski, Drug Discovery Today Technol. 2004, 1, 337 341; b) A. Lewandowicz, E. A. T. Ringia, L.-M. Ting, K. Kim, P. C. Tyler, G. B. Evans, O. V. Zubkova, S. Mee, G. F. Painter, 
D. H. Lenz, R. H. Furneaux, V. L. Schramm, J. Biol. Chem. 2005, 280, 30320-30328.

[20] T. K. Dam, C. F. Brewer, Adv. Carbohydr. Chem. Biochem., Vol. 63 (Ed.: H. Derek), Academic Press, 2010, pp. 139-164.

[21] M. Mammen, S.-K. Choi, G. M. Whitesides, Angew. Chem. Int. Ed. 1998, 37, 2754-2794; Angew. Chem. 1998, 110, 2908-2953.
[22] J. L. de Paz, C. Noti, F. Böhm, S. Werner, P. H. Seeberger, Chem. Biol. 2007, 14, 879-887.

[23] G. J. Sheng, Y. I. Oh, S.-K. Chang, L. C. Hsieh-Wilson, J. Am Chem. Soc. 2013, 135, 10898-10901.

[24] G. R. Newkome, X. Lin, Macromolecules 1991, 24, 1443-1444.

[25] R. P. Patel, S. Price, J. Org. Chem. 1965, 30, 3575-3576. 